\title{
A FIRST STUDY OF THE PYROLYSIS OF A HALOGENATED ESTER:
}

\section{METHYL CHLOROACETATE}

Nicolas Vin ${ }^{1,2}$, Frédérique Battin-Leclerc ${ }^{1}$, Olivier Herbinet ${ }^{1, *}$

${ }^{1}$ Laboratoire Réactions et Génie des Procédés, CNRS, Université de Lorraine, BP 20451, 1 rue Grandville, 54000 Nancy, France.

${ }^{2}$ Terbis, 943 rue Pasteur, 60700 Pont Sainte Maxence, France

*Corresponding author: olivier.herbinet@univ-lorraine.fr

\section{Supplementary Description}

1) Experimental setup

2) Temperature profiles in the TR

3) Carbon, oxygen and chlorine atom balances in the TR

4) SCF energy, zero-point corrected electronic energy, frequencies and geometry of molecules, radicals and transition states, calculated at the CBS-QB3 level of theory.

5) Comparison of the kinetic parameters of reaction R30 in Table 1 with literature data.

6) Experimental mole fractions of species in a tabular form 


\section{1) Experimental setup}

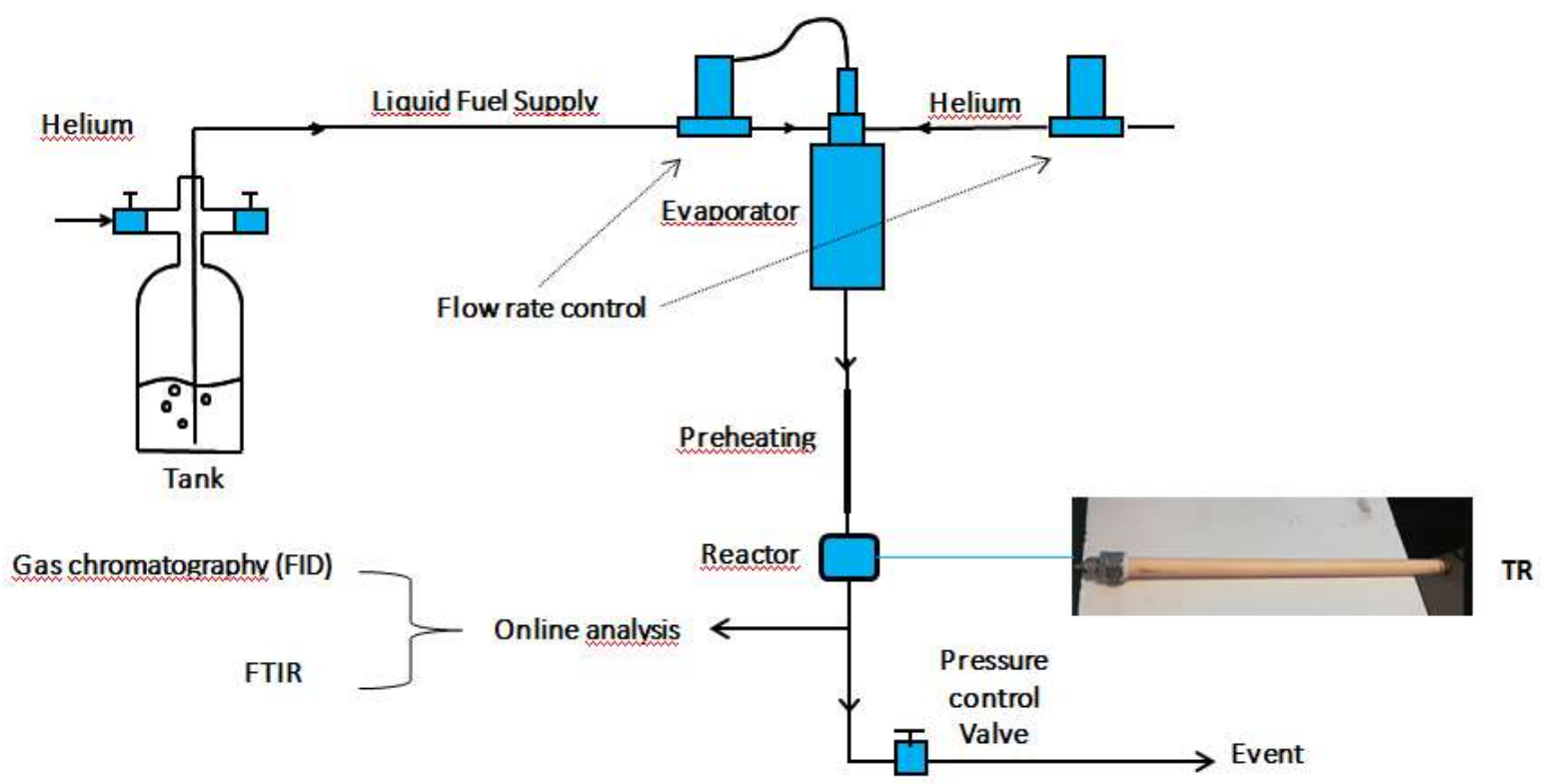

Figure S1: Scheme of the experimental setup used for running pyrolysis experiments.

\section{2) Temperature profiles in the TR}

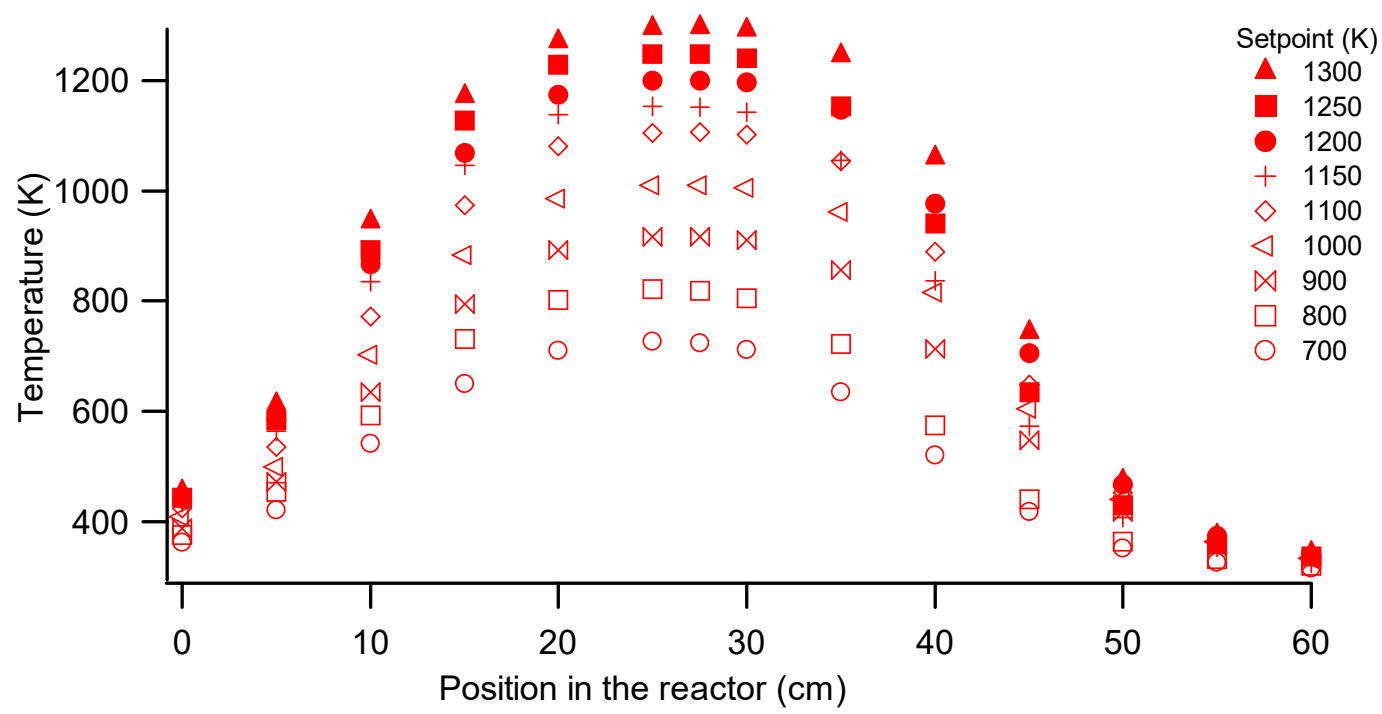

Figure S2 : Measured temperature profile in TR in absence of reaction. 


\section{3) Carbon, oxygen and chlorine atom balances in the TR}

The deviation was calculated as follow:

deviation $($ in $\%)=\frac{\text { number of atom } i \text { at the inlet }- \text { number of atom } i \text { at the outlet }}{\text { number of atom } i \text { at the inlet }} \times 100$

Table S1 : Carbon, oxygen and chlorine atom balances in the TR

\begin{tabular}{ccccc}
\hline $\mathbf{T}\left({ }^{\circ} \mathbf{C}\right)$ & $\mathbf{T}(\mathbf{K})$ & $\begin{array}{c}\text { Carbone } \\
\text { balance } \\
\mathbf{( \% )}\end{array}$ & $\begin{array}{c}\text { Oxygen } \\
\text { balance } \\
\mathbf{( \% )}\end{array}$ & $\begin{array}{c}\text { Chlorine } \\
\text { balance } \\
\mathbf{( \% )}\end{array}$ \\
\hline 300 & 573 & 0,0 & 0,0 & 0,0 \\
400 & 673 & 0,7 & 0,7 & 0,7 \\
450 & 723 & 7,3 & 7,3 & 7,3 \\
600 & 873 & $-4,2$ & $-4,6$ & $-3,8$ \\
650 & 923 & $-1,6$ & $-0,3$ & $-10,3$ \\
675 & 948 & 3,7 & 3,0 & $-7,1$ \\
700 & 973 & $-0,3$ & 0,5 & $-9,7$ \\
712 & 985 & 12,6 & 15,6 & 5,1 \\
725 & 998 & 9,8 & 13,2 & 9,9 \\
750 & 1023 & 14,7 & 17,5 & 25,6 \\
775 & 1048 & 7,3 & 6,5 & 27,3 \\
\hline
\end{tabular}


4) SCF energy, zero-point corrected electronic energy, frequencies and geometry of molecules, radicals and transition states, calculated at the CBS-QB3 level of theory.

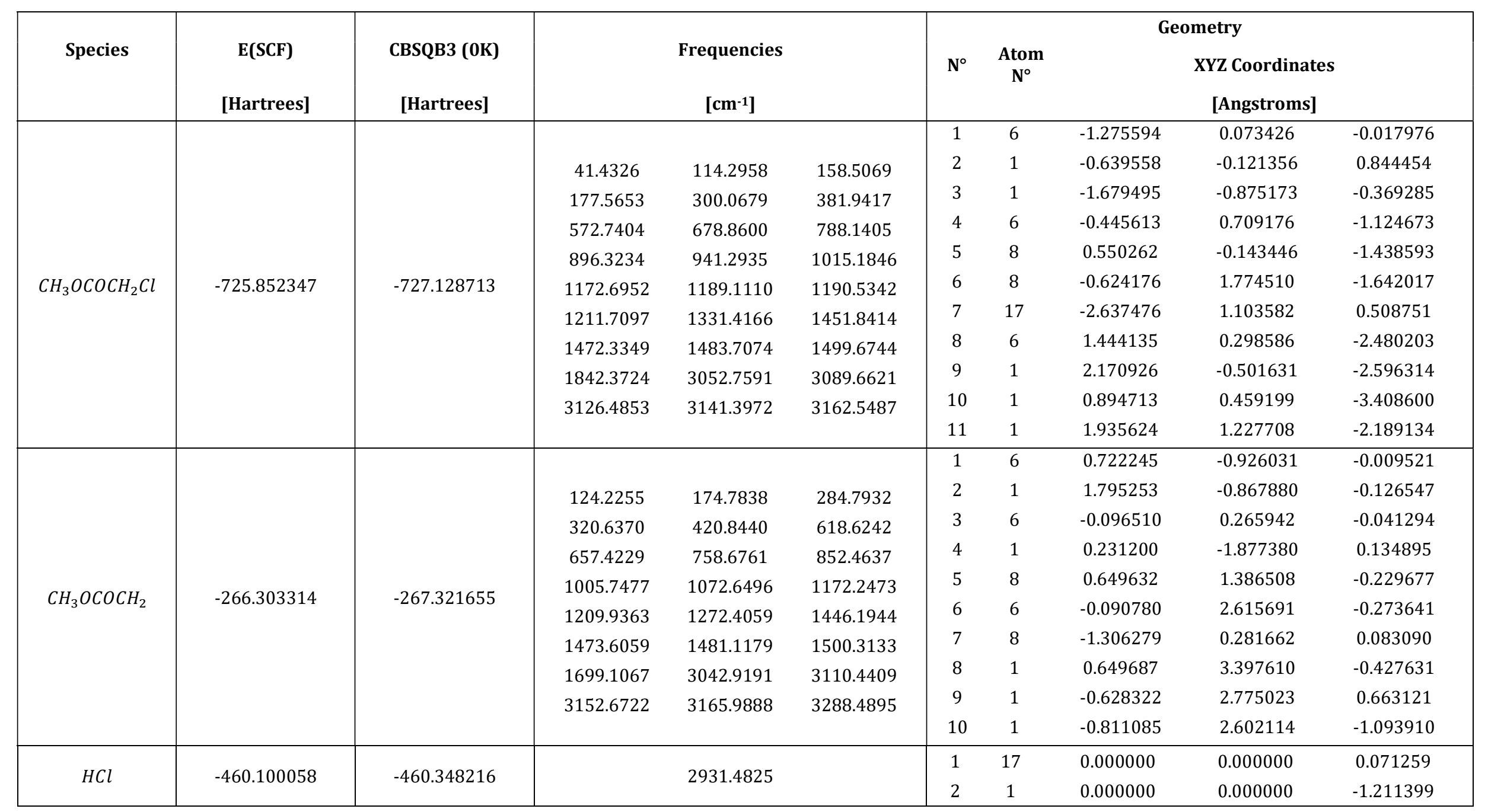




\begin{tabular}{|c|c|c|c|c|c|c|c|c|c|c|}
\hline \multirow{10}{*}{$\mathrm{CH}_{3} \mathrm{OCOCHCl}$} & \multirow{10}{*}{-725.231742} & \multirow{10}{*}{-726.484934} & & & & 1 & 6 & 0.314432 & 0.074057 & -0.004076 \\
\hline & & & 101.9264 & 129.1181 & 162.6581 & 2 & 1 & 0.033667 & -0.966813 & -0.054626 \\
\hline & & & 183.1055 & 312.5299 & 436.7165 & 3 & 6 & -0.694421 & 1.090218 & -0.240999 \\
\hline & & & 523.3051 & 664.3568 & 720.1359 & 4 & 8 & -0.233679 & 2.351794 & -0.154156 \\
\hline & & & 824.5856 & 934.1311 & 1070.8418 & 5 & 6 & -1.211891 & 3.381035 & -0.383872 \\
\hline & & & 1173.2861 & 1208.9293 & 1267.5389 & 6 & 8 & -1.845814 & 0.791939 & -0.494185 \\
\hline & & & 1329.9995 & 1472.8736 & 1482.6595 & 7 & 1 & -0.673724 & 4.319633 & -0.275556 \\
\hline & & & 1499.6754 & 1706.7768 & 3048.1333 & 8 & 1 & -2.018167 & 3.312302 & 0.348149 \\
\hline & & & 3118.7685 & 3160.0524 & 3241.7706 & 9 & 1 & -1.632866 & 3.291601 & -1.386596 \\
\hline & & & & & & 10 & 17 & 1.944212 & 0.412833 & 0.356491 \\
\hline \multirow{10}{*}{$\mathrm{CH}_{2} \mathrm{OCOCH}_{2} \mathrm{Cl}$} & \multirow{10}{*}{-725.218671} & \multirow{10}{*}{-726.471197} & & & & 1 & 6 & 1.351287 & -0.219589 & -0.020502 \\
\hline & & & 33.4425 & 95.3704 & 151.4535 & 2 & 1 & 1.215096 & -0.313075 & 1.056431 \\
\hline & & & 180.9379 & 290.7876 & 310.9951 & 3 & 6 & 0.280518 & 0.694862 & -0.587564 \\
\hline & & & 395.7732 & 562.1804 & 667.4933 & 4 & 8 & 0.398581 & 1.905340 & 0.044328 \\
\hline & & & 794.2732 & 874.7033 & 931.4086 & 5 & 6 & -0.472422 & 2.904391 & -0.296145 \\
\hline & & & 1095.4366 & 1177.6589 & 1187.1574 & 6 & 8 & -0.531652 & 0.448520 & -1.427305 \\
\hline & & & 1213.8239 & 1319.5547 & 1437.3521 & 7 & 1 & -0.284483 & 3.835525 & 0.209221 \\
\hline & & & 1450.9377 & 1850.0385 & 3085.4458 & 8 & 1 & -1.188350 & 2.721752 & -1.080181 \\
\hline & & & 3136.0499 & 3182.9098 & 3345.9364 & 9 & 17 & 1.312157 & -1.854302 & -0.738381 \\
\hline & & & & & & 10 & 1 & 2.333579 & 0.213912 & -0.206467 \\
\hline \multirow{2}{*}{$\mathrm{H}_{2}$} & \multirow{2}{*}{-1.132955} & \multirow{2}{*}{-1.166083} & \multirow{2}{*}{\multicolumn{3}{|c|}{4419.2598}} & 1 & 1 & -0.730352 & 0.274390 & 0.000000 \\
\hline & & & & & & 2 & 1 & -1.474526 & 0.274390 & 0.000000 \\
\hline \multirow{4}{*}{$\mathrm{CH}_{3}$} & \multirow{4}{*}{-39.576435} & \multirow{4}{*}{-39.744796} & & & & 1 & 6 & -2.055314 & -0.629625 & 0.000001 \\
\hline & & & 505.1758 & 1403.1570 & 1403.1712 & 2 & 1 & -2.056890 & -1.710074 & 0.000004 \\
\hline & & & 3104.1993 & 3283.2781 & 3283.3235 & 3 & 1 & -2.056891 & -0.089411 & -0.935704 \\
\hline & & & & & & 4 & 1 & -2.054447 & -0.089413 & 0.935709 \\
\hline \multirow{5}{*}{$\mathrm{CH}_{4}$} & \multirow{5}{*}{-40.355896} & \multirow{5}{*}{-40.465297} & & & & 1 & 6 & 0.000000 & 0.000000 & 0.000000 \\
\hline & & & 1341.6165 & 1341.6165 & 1341.6165 & 2 & 1 & 0.630364 & 0.630364 & 0.630364 \\
\hline & & & 1563.5772 & 1563.5772 & 3039.2754 & 3 & 1 & -0.630364 & -0.630364 & 0.630364 \\
\hline & & & 3153.2315 & 3153.2315 & 3153.2315 & 4 & 1 & -0.630364 & 0.630364 & -0.630364 \\
\hline & & & & & & 5 & 1 & 0.630364 & -0.630364 & -0.630364 \\
\hline
\end{tabular}




\begin{tabular}{|c|c|c|c|c|c|c|c|c|c|c|}
\hline \multirow{5}{*}{$\mathrm{CH}_{3} \mathrm{Cl}$} & \multirow{5}{*}{-499.139907} & \multirow{5}{*}{-499.562037} & & & & 1 & 6 & -0.602940 & 0.697406 & 0.000000 \\
\hline & & & 708.2450 & 1026.1341 & 1026.4132 & 2 & 1 & -0.229271 & 1.189408 & 0.894333 \\
\hline & & & 1381.4430 & 1479.8686 & 1479.9441 & 3 & 1 & -0.229271 & 1.189408 & -0.894333 \\
\hline & & & 3072.7596 & 3171.7106 & 3172.0424 & 4 & 1 & -1.689624 & 0.673181 & 0.000000 \\
\hline & & & & & & 5 & 17 & -0.001808 & -1.002827 & 0.000000 \\
\hline \multirow{6}{*}{$\mathrm{COCH}_{2} \mathrm{Cl}$} & \multirow{6}{*}{-611.275124} & \multirow{6}{*}{-612.095201} & & & & 1 & 6 & -0.352822 & 0.884316 & -0.007512 \\
\hline & & & 174.7089 & 216.4721 & 573.7920 & 2 & 8 & -0.892512 & 1.004380 & -1.041667 \\
\hline & & & 647.4199 & 789.3375 & 829.4286 & 3 & 6 & 0.147175 & -0.295700 & 0.756767 \\
\hline & & & 1180.3678 & 1258.4000 & 1415.3662 & 4 & 17 & -0.106086 & -1.927245 & -0.037281 \\
\hline & & & 1972.9222 & 3090.0306 & 3144.7702 & 5 & 1 & 1.217640 & -0.171840 & 0.919894 \\
\hline & & & & & & 6 & 1 & -0.356608 & -0.318242 & 1.722955 \\
\hline \multirow{4}{*}{$\mathrm{CH}_{2} \mathrm{Cl}$} & \multirow{4}{*}{-498.507950} & \multirow{4}{*}{-498.905997} & & & & 1 & 6 & 1.759770 & 0.677247 & -0.258610 \\
\hline & & & 155.8240 & 824.7556 & 996.2595 & 2 & 1 & 2.322682 & 1.390308 & -0.838369 \\
\hline & & & 1409.1347 & 3167.9616 & 3324.1861 & 3 & 1 & 0.764472 & 0.840168 & 0.121236 \\
\hline & & & & & & 4 & 17 & 2.499756 & -0.825262 & 0.081410 \\
\hline \multirow{2}{*}{$\mathrm{CO}$} & \multirow{2}{*}{-112.777311} & \multirow{2}{*}{-113.182057} & \multirow{2}{*}{\multicolumn{3}{|c|}{2219.9667}} & 1 & 6 & -0.779342 & 0.187113 & -0.004865 \\
\hline & & & & & & 2 & 8 & -1.271775 & 0.011330 & -1.003174 \\
\hline \multirow{12}{*}{$T S 1^{* *}$} & \multirow{12}{*}{-726.324795} & \multirow{12}{*}{-727.614442} & & & & 1 & 6 & -1.489829 & -0.038214 & -0.008542 \\
\hline & & & -785.3707 & 51.9075 & 106.3150 & 2 & 1 & -1.335565 & 0.420420 & 0.961125 \\
\hline & & & 110.6546 & 122.7757 & 155.9199 & 3 & 1 & -1.587640 & -1.118299 & 0.028504 \\
\hline & & & 222.5019 & 277.5717 & 407.1537 & 4 & 6 & -0.469110 & 0.437397 & -1.005216 \\
\hline & & & 588.9840 & 678.4753 & 770.4773 & 5 & 8 & -0.453669 & -0.350933 & -2.093074 \\
\hline & & & 845.0227 & 913.3711 & 1027.5802 & 6 & 8 & 0.241829 & 1.396318 & -0.842846 \\
\hline & & & 1102.1380 & 1173.1823 & 1196.3540 & 7 & 6 & 0.457998 & 0.044741 & -3.136212 \\
\hline & & & 1212.9808 & 1286.4955 & 1453.7595 & 8 & 1 & 0.325921 & -0.687455 & -3.928922 \\
\hline & & & 1474.7273 & 1483.6248 & 1497.9867 & 9 & 1 & 0.213854 & 1.046725 & -3.491606 \\
\hline & & & 1802.0831 & 3051.4606 & 3124.1738 & 10 & 1 & 1.484846 & 0.035409 & -2.767977 \\
\hline & & & 3130.0706 & 3162.7544 & 3219.7288 & 11 & 1 & -4.940599 & 1.037429 & -0.865880 \\
\hline & & & & & & 12 & 17 & -3.222025 & 0.574847 & -0.569925 \\
\hline
\end{tabular}




\begin{tabular}{|c|c|c|c|c|c|c|c|c|c|c|}
\hline \multirow{12}{*}{$T S 2^{* *}$} & \multirow{12}{*}{-726.319354} & \multirow{12}{*}{-727.616333} & & & & 1 & 6 & -0.166960 & -0.278983 & 0.025663 \\
\hline & & & -1320.2069 & 59.2646 & 106.0586 & 2 & 1 & -0.411954 & -1.315830 & -0.188554 \\
\hline & & & 149.2749 & 183.3656 & 294.1167 & 3 & 6 & 0.667217 & 0.373209 & -1.046060 \\
\hline & & & 307.6654 & 339.5198 & 394.4756 & 4 & 8 & 1.559004 & -0.530108 & -1.498866 \\
\hline & & & 669.0082 & 691.5603 & 815.5799 & 5 & 8 & 0.591232 & 1.512470 & -1.415850 \\
\hline & & & 901.6592 & 959.1163 & 1022.5029 & 6 & 17 & -1.595015 & 0.626282 & 0.501413 \\
\hline & & & 1167.6262 & 1172.9330 & 1194.8450 & 7 & 6 & 2.490151 & -0.042972 & -2.486549 \\
\hline & & & 1212.1004 & 1315.1264 & 1327.8344 & 8 & 1 & 3.132434 & -0.888569 & -2.719769 \\
\hline & & & 1347.8594 & 1471.6984 & 1482.8690 & 9 & 1 & 1.956609 & 0.294071 & -3.376100 \\
\hline & & & 1498.6230 & 1821.7578 & 3052.5444 & 10 & 1 & 3.072976 & 0.785486 & -2.082004 \\
\hline & & & 3126.1886 & 3147.5294 & 3163.3358 & 11 & 1 & 1.179630 & -0.457463 & 1.866230 \\
\hline & & & & & & 12 & 1 & 0.579712 & -0.357031 & 1.031859 \\
\hline \multirow{12}{*}{$T S 3^{* *}$} & \multirow{12}{*}{-726.314606} & \multirow{12}{*}{-727.611183} & & & & 1 & 6 & -1.346989 & 0.721257 & -0.002375 \\
\hline & & & -1315.8873 & 44.4661 & 93.1432 & 2 & 1 & -0.727324 & 0.250586 & 0.759801 \\
\hline & & & 138.7443 & 182.0936 & 309.6166 & 3 & 1 & -1.892765 & -0.058676 & -0.532640 \\
\hline & & & 320.1137 & 398.1265 & 546.3679 & 4 & 6 & -0.460858 & 1.466637 & -0.986188 \\
\hline & & & 567.7184 & 679.5196 & 793.2656 & 5 & 8 & 0.435331 & 0.571455 & -1.491503 \\
\hline & & & 886.3695 & 937.1358 & 1054.5870 & 6 & 8 & -0.509170 & 2.621750 & -1.285521 \\
\hline & & & 1109.0626 & 1164.6728 & 1190.5183 & 7 & 17 & -2.530012 & 1.786323 & 0.806741 \\
\hline & & & 1199.0943 & 1271.2699 & 1283.4654 & 8 & 6 & 1.331021 & 1.040426 & -2.464043 \\
\hline & & & 1326.9693 & 1449.9538 & 1454.5653 & 9 & 1 & 2.162235 & 0.346293 & -2.538408 \\
\hline & & & 1519.3978 & 1852.0135 & 3087.7412 & 10 & 1 & 1.588572 & 2.090399 & -2.348618 \\
\hline & & & 3098.1560 & 3139.3105 & 3208.7339 & 11 & 1 & 0.267754 & 0.915609 & -4.485363 \\
\hline & & & & & & 12 & 1 & 0.728848 & 0.978798 & -3.648535 \\
\hline
\end{tabular}




\begin{tabular}{|c|c|c|c|c|c|c|c|c|c|c|}
\hline \multirow{15}{*}{$T S 4^{* *}$} & \multirow{15}{*}{-765.385214} & \multirow{15}{*}{-766.851062} & & & & 1 & 6 & -0.365700 & 0.164910 & -0.000713 \\
\hline & & & -497.9081 & 37.8819 & 47.9494 & 2 & 1 & -0.098793 & 0.158296 & 1.047801 \\
\hline & & & 59.8218 & 92.1782 & 113.4940 & 3 & 1 & -0.778424 & -0.756753 & -0.391607 \\
\hline & & & 133.1146 & 194.3760 & 281.0710 & 4 & 6 & 0.641266 & 0.836155 & -0.860006 \\
\hline & & & 356.3835 & 411.7001 & 652.8769 & 5 & 8 & 0.452529 & 0.532397 & -2.162443 \\
\hline & & & 657.8207 & 661.4368 & 744.9410 & 6 & 8 & 1.516159 & 1.564715 & -0.456242 \\
\hline & & & 791.3675 & 876.3097 & 1018.3677 & 7 & 6 & 1.352846 & 1.173320 & -3.081804 \\
\hline & & & 1034.5514 & 1064.8456 & 1078.5948 & 8 & 1 & 1.055431 & 0.827760 & -4.069258 \\
\hline & & & 1172.9892 & 1210.5327 & 1270.8506 & 9 & 1 & 1.261957 & 2.258449 & -3.010268 \\
\hline & & & 1427.8759 & 1428.4319 & 1449.4914 & 10 & 1 & 2.384810 & 0.889113 & -2.868979 \\
\hline & & & 1474.2125 & 1482.8322 & 1499.3384 & 11 & 6 & -3.865235 & 2.614562 & 0.101874 \\
\hline & & & 1778.7649 & 3046.6339 & 3097.6616 & 12 & 1 & -4.390425 & 2.150751 & 0.926561 \\
\hline & & & 3116.6896 & 3148.5296 & 3156.3651 & 13 & 1 & -3.439034 & 3.591185 & 0.290742 \\
\hline & & & 3250.1660 & 3257.8780 & 3258.3902 & 14 & 1 & -4.277574 & 2.450408 & -0.885168 \\
\hline & & & & & & 15 & 17 & -2.046539 & 1.384152 & 0.006692 \\
\hline \multirow{15}{*}{$T S 5^{* *}$} & \multirow{15}{*}{-765.388977} & \multirow{15}{*}{-766.860103} & & & & 1 & 6 & -0.190582 & -0.001836 & -0.002017 \\
\hline & & & -1504.6707 & 41.8900 & 49.4536 & 2 & 1 & -0.785363 & -0.903555 & -0.117385 \\
\hline & & & 75.0046 & 114.3828 & 126.2223 & 3 & 6 & 0.715598 & 0.268171 & -1.164222 \\
\hline & & & 177.3669 & 194.2284 & 301.1334 & 4 & 8 & 1.203785 & -0.912504 & -1.610392 \\
\hline & & & 384.1527 & 441.5660 & 514.7643 & 5 & 8 & 1.019078 & 1.342209 & -1.610048 \\
\hline & & & 563.2960 & 703.0711 & 722.2301 & 6 & 17 & -1.201600 & 1.361363 & 0.475167 \\
\hline & & & 816.6491 & 910.9654 & 987.4951 & 7 & 6 & 2.155337 & -0.820305 & -2.687620 \\
\hline & & & 1032.4815 & 1124.9792 & 1170.9241 & 8 & 1 & 2.438432 & -1.845802 & -2.913163 \\
\hline & & & 1173.6880 & 1209.4009 & 1278.1917 & 9 & 1 & 1.699712 & -0.346184 & -3.557979 \\
\hline & & & 1366.0685 & 1378.5349 & 1428.4032 & 10 & 1 & 3.025685 & -0.238791 & -2.379756 \\
\hline & & & 1429.2716 & 1471.3686 & 1482.8747 & 11 & 6 & 1.409285 & -0.568538 & 2.111154 \\
\hline & & & 1499.8474 & 1812.6470 & 3049.8279 & 12 & 1 & 1.983641 & 0.345842 & 2.226929 \\
\hline & & & 3067.4101 & 3121.8142 & 3149.7540 & 13 & 1 & 0.689368 & -0.762322 & 2.900514 \\
\hline & & & 3158.6009 & 3213.3139 & 3214.0364 & 14 & 1 & 1.974316 & -1.432742 & 1.774817 \\
\hline & & & & & & 15 & 1 & 0.578550 & -0.264290 & 0.989866 \\
\hline
\end{tabular}




\begin{tabular}{|c|c|c|c|c|c|c|c|c|c|c|}
\hline \multirow{15}{*}{$T S 6^{* *}$} & \multirow{15}{*}{-765.381715} & \multirow{15}{*}{-766.852294} & & & & 1 & 6 & -0.659987 & -0.078005 & 0.000692 \\
\hline & & & -1647.4526 & 24.0904 & 47.7518 & 2 & 1 & -0.630807 & -1.156972 & 0.146474 \\
\hline & & & 60.3112 & 69.0554 & 177.8952 & 3 & 1 & -1.559744 & 0.169699 & -0.561397 \\
\hline & & & 186.4028 & 304.4491 & 336.2418 & 4 & 6 & 0.565197 & 0.358356 & -0.788745 \\
\hline & & & 380.4287 & 488.1217 & 552.8363 & 5 & 8 & 0.556287 & -0.313174 & -1.966239 \\
\hline & & & 601.5253 & 660.1154 & 709.2085 & 6 & 8 & 1.397575 & 1.152368 & -0.459234 \\
\hline & & & 791.4701 & 894.2792 & 936.8594 & 7 & 17 & -0.767123 & 0.704525 & 1.603821 \\
\hline & & & 1044.9596 & 1143.0694 & 1156.5407 & 8 & 6 & 1.623355 & -0.057742 & -2.860079 \\
\hline & & & 1186.7282 & 1191.3168 & 1234.2291 & 9 & 1 & 1.346318 & -0.457680 & -3.831142 \\
\hline & & & 1325.2767 & 1387.0867 & 1403.2379 & 10 & 1 & 1.931180 & 0.985864 & -2.859787 \\
\hline & & & 1441.5463 & 1445.6776 & 1450.4527 & 11 & 6 & 3.763743 & -1.441946 & -1.960133 \\
\hline & & & 1468.2608 & 1839.9222 & 3058.6215 & 12 & 1 & 3.406649 & -2.464520 & -1.864467 \\
\hline & & & 3087.7410 & 3089.7438 & 3139.7357 & 13 & 1 & 4.538468 & -1.313353 & -2.712240 \\
\hline & & & 3190.5851 & 3194.5390 & 3198.0564 & 14 & 1 & 3.981219 & -0.955009 & -1.013304 \\
\hline & & & & & & 15 & 1 & 2.679489 & -0.736662 & -2.450682 \\
\hline \multirow{10}{*}{$T S 7^{* *}$} & \multirow{10}{*}{-725.153366} & \multirow{10}{*}{-726.431030} & & & & 1 & 6 & -0.890982 & 0.654510 & -0.012210 \\
\hline & & & -1755.0658 & 66.7827 & 162.0636 & 2 & 1 & -1.366725 & -0.203533 & 0.458785 \\
\hline & & & 209.0958 & 413.6509 & 500.8508 & 3 & 6 & -1.538751 & 1.068041 & -1.327631 \\
\hline & & & 572.6063 & 623.4435 & 693.2730 & 4 & 8 & -2.350475 & 2.154759 & -1.116838 \\
\hline & & & 814.4165 & 884.9661 & 943.4130 & 5 & 6 & -2.262115 & 2.671084 & 0.190320 \\
\hline & & & 1004.5092 & 1094.3089 & 1166.8028 & 6 & 8 & -1.409643 & 0.558194 & -2.394305 \\
\hline & & & 1175.3448 & 1242.8600 & 1390.6610 & 7 & 1 & -1.812363 & 3.660984 & 0.217886 \\
\hline & & & 1484.8060 & 1724.9701 & 1891.4471 & 8 & 1 & -1.326664 & 1.735840 & 0.620972 \\
\hline & & & 3082.2710 & 3129.2766 & 3186.3001 & 9 & 1 & -3.181481 & 2.546531 & 0.757787 \\
\hline & & & & & & 10 & 17 & 0.849568 & 0.445246 & -0.064977 \\
\hline
\end{tabular}




\begin{tabular}{|c|c|c|c|c|c|c|c|c|c|c|}
\hline \multirow{10}{*}{$T S 8^{* *}$} & \multirow{10}{*}{-725.152572} & \multirow{10}{*}{-726.423620} & & & & 1 & 6 & -0.061971 & 1.843812 & 0.000743 \\
\hline & & & -545.9469 & 57.2806 & 135.6529 & 2 & 1 & -0.776077 & 1.031305 & -0.119995 \\
\hline & & & 202.5468 & 221.1492 & 304.5695 & 3 & 6 & -0.407487 & 2.917824 & -0.938137 \\
\hline & & & 403.8114 & 501.0335 & 510.0091 & 4 & 8 & 0.237349 & 4.403156 & -0.023281 \\
\hline & & & 605.1684 & 863.6161 & 910.4591 & 5 & 6 & 0.475351 & 5.376697 & -0.748300 \\
\hline & & & 1032.8824 & 1173.2631 & 1235.3422 & 6 & 8 & -0.371478 & 3.085025 & -2.110265 \\
\hline & & & 1248.7151 & 1421.5807 & 1438.9441 & 7 & 1 & 0.551784 & 5.249510 & -1.834030 \\
\hline & & & 1575.4873 & 1890.0407 & 3013.1061 & 8 & 1 & 0.573429 & 6.369872 & -0.302590 \\
\hline & & & 3113.8272 & 3126.6900 & 3201.6426 & 9 & 17 & 1.614771 & 1.057168 & -0.307816 \\
\hline & & & & & & 10 & 1 & -0.037037 & 2.205295 & 1.021869 \\
\hline \multirow{6}{*}{$T S 9 * *$} & \multirow{6}{*}{-611.259683} & \multirow{6}{*}{-612.074460} & & & & 1 & 6 & -0.843213 & 0.159740 & 0.011311 \\
\hline & & & -336.3080 & 65.9764 & 117.5353 & 2 & 8 & -1.344131 & -0.005599 & -0.999551 \\
\hline & & & 349.2776 & 545.3971 & 752.1996 & 3 & 6 & -0.142486 & -1.578982 & 1.066662 \\
\hline & & & 937.9691 & 1046.2653 & 1413.9038 & 4 & 17 & -0.454532 & -3.041410 & 0.188508 \\
\hline & & & 2082.4778 & 3131.9626 & 3258.7063 & 5 & 1 & 0.905955 & -1.316054 & 1.120599 \\
\hline & & & & & & 6 & 1 & -0.741660 & -1.469407 & 1.961221 \\
\hline \multirow{12}{*}{$T S 10^{* *}$} & \multirow{12}{*}{-1185.293496} & \multirow{12}{*}{-1186.817684} & & & & 1 & 6 & 0.097609 & -0.036669 & 0.004684 \\
\hline & & & -967.6139 & 42.6637 & 62.7187 & 2 & 1 & 0.902688 & -0.227909 & 0.707791 \\
\hline & & & 109.5297 & 122.0990 & 149.7613 & 3 & 6 & 0.591753 & 0.390124 & -1.352118 \\
\hline & & & 194.2565 & 300.2245 & 370.2901 & 4 & 8 & -0.382676 & 0.503982 & -2.248058 \\
\hline & & & 443.7855 & 621.9509 & 706.7267 & 5 & 8 & 1.760655 & 0.618050 & -1.537508 \\
\hline & & & 820.8391 & 887.0549 & 908.0244 & 6 & 17 & -1.149010 & -1.240936 & 0.081924 \\
\hline & & & 984.3081 & 1044.5658 & 1142.4528 & 7 & 6 & 0.019676 & 0.948711 & -3.563027 \\
\hline & & & 1173.0979 & 1210.7508 & 1269.5790 & 8 & 1 & -0.899501 & 1.000224 & -4.139871 \\
\hline & & & 1292.7647 & 1474.6408 & 1485.3813 & 9 & 1 & 0.493183 & 1.928537 & -3.496805 \\
\hline & & & 1495.3738 & 1782.9981 & 3057.2955 & 10 & 1 & 0.715889 & 0.234815 & -4.004024 \\
\hline & & & 3133.9593 & 3169.5893 & 3173.7996 & 11 & 1 & -0.429388 & 1.081102 & 0.498719 \\
\hline & & & & & & 12 & 17 & -0.923076 & 2.389898 & 1.122691 \\
\hline
\end{tabular}




\begin{tabular}{|c|c|c|c|c|c|c|c|c|c|c|}
\hline \multirow{12}{*}{$T S 11^{* *}$} & \multirow{12}{*}{-1185.297764} & \multirow{12}{*}{-1186.816397} & & & & 1 & 6 & -0.435465 & 1.000573 & 0.002828 \\
\hline & & & -702.8621 & 30.3833 & 47.6144 & 2 & 1 & 0.115997 & 0.353435 & 0.683758 \\
\hline & & & 57.1110 & 180.5524 & 187.1926 & 3 & 1 & -1.047701 & 0.374010 & -0.646845 \\
\hline & & & 312.6404 & 387.9509 & 410.9552 & 4 & 6 & 0.538297 & 1.780577 & -0.853204 \\
\hline & & & 534.0788 & 558.2673 & 668.0225 & 5 & 8 & 1.417956 & 0.864128 & -1.416753 \\
\hline & & & 796.9612 & 856.8942 & 927.5284 & 6 & 8 & 0.590161 & 2.951498 & -1.050433 \\
\hline & & & 933.0008 & 962.1832 & 1082.1107 & 7 & 17 & -1.511598 & 2.057204 & 0.953289 \\
\hline & & & 1173.1579 & 1187.7184 & 1194.0440 & 8 & 6 & 2.316561 & 1.328264 & -2.341984 \\
\hline & & & 1232.5273 & 1324.4251 & 1445.5980 & 9 & 1 & 3.147485 & 0.641213 & -2.457310 \\
\hline & & & 1448.9981 & 1876.4978 & 3083.7041 & 10 & 1 & 2.500286 & 2.398857 & -2.321114 \\
\hline & & & 3105.1062 & 3136.1299 & 3235.2350 & 11 & 1 & 1.698388 & 1.189175 & -3.638688 \\
\hline & & & & & & 12 & 17 & 1.052026 & 1.022148 & -4.933098 \\
\hline
\end{tabular}

* Transition state and associated reactions:

TS1: $\mathrm{CH}_{3} \mathrm{OCOCH}_{2} \mathrm{Cl}+\mathrm{H}=\mathrm{CH}_{3} \mathrm{OCOCH}_{2}+\mathrm{HCl}$

TS2: $\mathrm{CH}_{3} \mathrm{OCOCH}_{2} \mathrm{Cl}+\mathrm{H}=\mathrm{CH}_{3} \mathrm{OCOCHCl}+\mathrm{H}_{2}$

TS3: $\mathrm{CH}_{3} \mathrm{OCOCH}_{2} \mathrm{Cl}+\mathrm{H}=\mathrm{CH}_{2} \mathrm{OCOCH}_{2} \mathrm{Cl}+\mathrm{H}_{2}$

TS4: $\mathrm{CH}_{3} \mathrm{OCOCH}_{2} \mathrm{Cl}+\mathrm{CH}_{3}=\mathrm{CH}_{3} \mathrm{OCOCH}_{2}+\mathrm{CH}_{3} \mathrm{Cl}$

TS5: $\mathrm{CH}_{3} \mathrm{OCOCH}_{2} \mathrm{Cl}+\mathrm{CH}_{3}=\mathrm{CH}_{3} \mathrm{OCOCHCl}+\mathrm{CH}_{4}$

TS6: $\mathrm{CH}_{3} \mathrm{OCOCH}_{2} \mathrm{Cl}+\mathrm{CH}_{3}=\mathrm{CH}_{2} \mathrm{OCOCH}_{2} \mathrm{Cl}+\mathrm{CH}_{4}$

TS7: $\mathrm{CH}_{3} \mathrm{OCOCHCl}=\mathrm{CH}_{2} \mathrm{OCOCH}_{2} \mathrm{Cl}$

TS8: $\mathrm{CH}_{2} \mathrm{OCOCH}_{2} \mathrm{Cl}=\mathrm{CH}_{2} \mathrm{O}+\mathrm{COCH}_{2} \mathrm{Cl}$

TS9: $\mathrm{COCH}_{2} \mathrm{Cl}=\mathrm{CO}+\mathrm{CH}_{2} \mathrm{Cl}$

TS10: $\mathrm{CH}_{3} \mathrm{OCOCH}_{2} \mathrm{Cl}+\mathrm{Cl}=\mathrm{CH}_{3} \mathrm{OCOCHCl}+\mathrm{HCl}$

TS11: $\mathrm{CH}_{3} \mathrm{OCOCH}_{2} \mathrm{Cl}+\mathrm{Cl}=\mathrm{CH}_{2} \mathrm{OCOCH}_{2} \mathrm{Cl}+\mathrm{HCl}$ 
5) Comparison of the kinetic parameters of reaction R30 in Table 1 with literature data.

Figure $\mathrm{S} 1$ displays the comparison of the kinetic constant calculated for reaction $\mathrm{R} 30 \mathrm{COCH}_{2} \mathrm{Cl}$ $=\mathrm{CO}+\mathrm{CH}_{2} \mathrm{Cl}$ ) with kinetic constants calculated at different level of theory by Mereau et al. (Mereau et al., 2001). Kinetic parameters are given in Table S1.

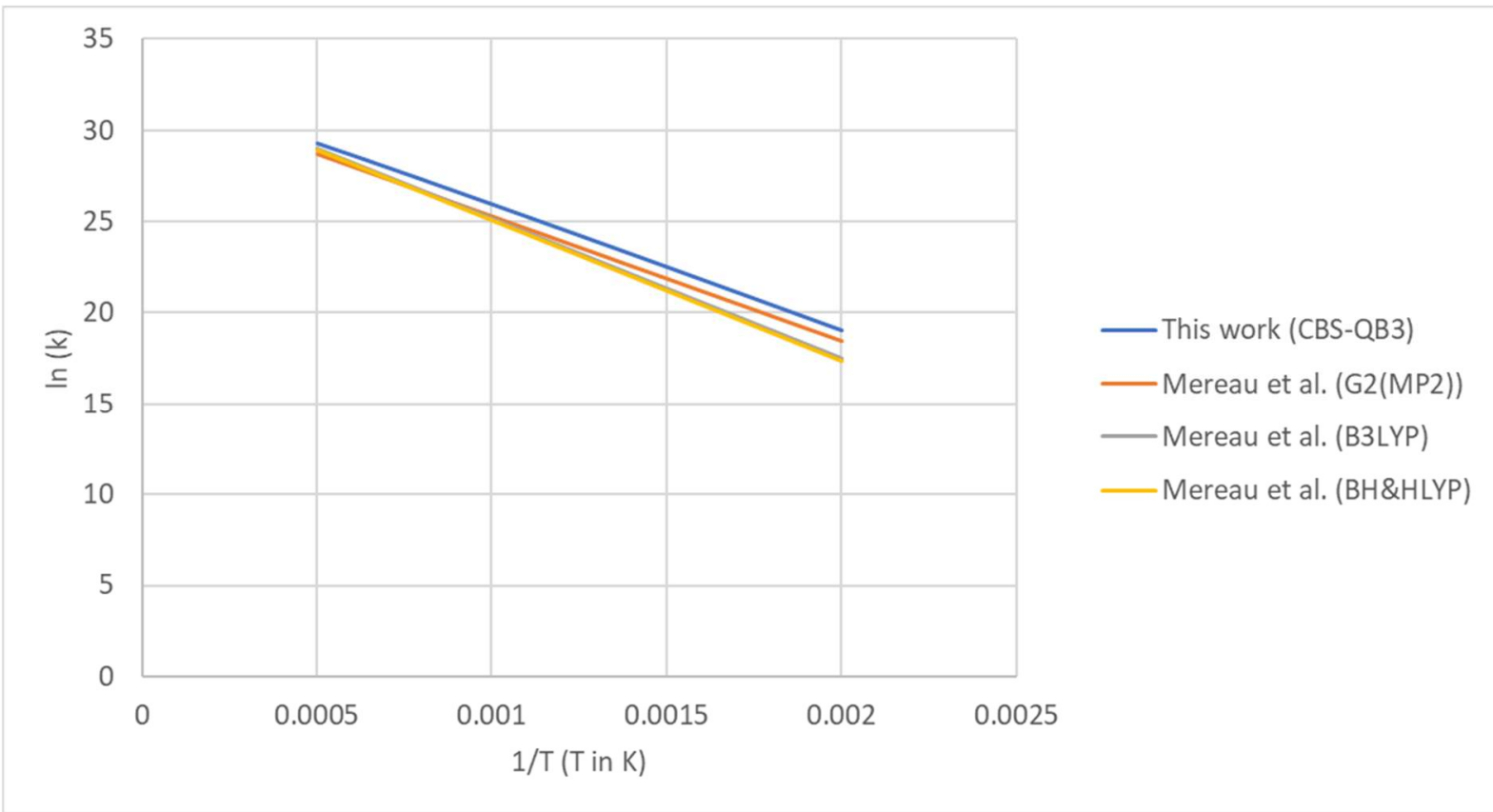

Figure S1: Comparison of the kinetic constant calculated for reaction $\mathrm{R} 30\left(\mathrm{COCH}_{2} \mathrm{Cl}=\mathrm{CO}+\right.$ $\mathrm{CH}_{2} \mathrm{Cl}$ ) with kinetic constants calculated at different level of theory by Mereau et al. (Mereau et al., 2001).

Table S1: Kinetic parameters of reaction R30.

\begin{tabular}{ccccc} 
& this work & \multicolumn{4}{c}{ Mereau et al., 2001} \\
\hline $\mathrm{A}\left(\mathrm{s}^{-1}\right)$ & $2.32 \mathrm{E}+15$ & $9.20 \mathrm{E}+13$ & $1.80 \mathrm{E}+14$ & $1.70 \mathrm{E}+14$ \\
$\mathrm{n}$ & -0.33 & & & \\
$\mathrm{Ea}(\mathrm{cal} / \mathrm{mol})$ & 14186 & 13599 & 15201 & 15296 \\
\hline
\end{tabular}

Mereau, R., Rayez, M.T., Rayez, J.C., Caralp, F., Lesclaux, R., 2001. Theoretical study on the atmospheric fate of carbonyl radicals: kinetics of decomposition reactions. Phys. Chem. Chem. Phys. 3, 4712-4717. https://doi.org/10.1039/b105824j 


\section{6) Experimental mole fractions of species in a tabular form}

$x_{\text {inlet }}=0.01$

residence time $=2 \mathrm{~s}$

$\mathrm{P}=1.067 \mathrm{bar}$

\begin{tabular}{|c|c|c|c|c|c|c|c|c|c|c|c|}
\hline \multirow[b]{2}{*}{$\mathbf{T}(\mathbf{K})$} & \multicolumn{11}{|c|}{ GC Data } \\
\hline & $\begin{array}{c}\text { Methyl } \\
\text { chloroacetate }\end{array}$ & $\begin{array}{c}\text { Chlorometha } \\
\text { ne } \\
\end{array}$ & $\begin{array}{c}\text { Chloroethyle } \\
\text { ne }\end{array}$ & $\begin{array}{c}\begin{array}{c}\text { Dichloromet } \\
\text { hane }\end{array} \\
\end{array}$ & Methane & Ethylene & Ethane & Propene & $\begin{array}{c}1,3- \\
\text { butadiene }\end{array}$ & $\begin{array}{c}\text { Methyl } \\
\text { formate }\end{array}$ & Acetic acid \\
\hline 573 & $1.00 \mathrm{E}-02$ & 0 & 0 & 0 & 0 & 0 & 0 & 0 & 0 & 0 & 0 \\
\hline 673 & $9.93 \mathrm{E}-03$ & 0 & 0 & 0 & 0 & 0 & 0 & 0 & 0 & 0 & 0 \\
\hline 723 & $9.27 \mathrm{E}-03$ & 0 & 0 & 0 & 0 & 0 & 0 & 0 & 0 & 0 & 0 \\
\hline 873 & $1.02 \mathrm{E}-02$ & 8.99E-05 & $1.11 \mathrm{E}-05$ & 0 & $3.33 \mathrm{E}-05$ & $4.96 \mathrm{E}-06$ & 0 & 0 & 0 & 0 & 0 \\
\hline 923 & 8.93E-03 & $6.21 \mathrm{E}-04$ & $1.27 \mathrm{E}-04$ & $3.77 \mathrm{E}-05$ & 4.12E-04 & $7.71 \mathrm{E}-05$ & $3.62 \mathrm{E}-06$ & 0 & 0 & 0 & 0 \\
\hline 948 & $7.27 \mathrm{E}-03$ & $9.21 \mathrm{E}-04$ & $2.01 \mathrm{E}-04$ & $4.20 \mathrm{E}-05$ & $5.94 \mathrm{E}-04$ & $1.29 \mathrm{E}-04$ & $5.44 \mathrm{E}-06$ & $3.42 \mathrm{E}-06$ & 0 & 0 & 0 \\
\hline 973 & $5.47 \mathrm{E}-03$ & $2.05 \mathrm{E}-03$ & $3.82 \mathrm{E}-04$ & 4.93E-05 & $1.31 \mathrm{E}-03$ & $3.38 \mathrm{E}-04$ & $1.38 \mathrm{E}-05$ & $1.00 \mathrm{E}-05$ & 0 & $3.09 \mathrm{E}-05$ & 0 \\
\hline 985 & $2.84 \mathrm{E}-03$ & $2.67 \mathrm{E}-03$ & $4.56 \mathrm{E}-04$ & $3.77 \mathrm{E}-05$ & $1.92 \mathrm{E}-03$ & $6.39 \mathrm{E}-04$ & $1.99 \mathrm{E}-05$ & $1.30 \mathrm{E}-05$ & $4.01 \mathrm{E}-06$ & 7.34E-05 & $2.46 \mathrm{E}-05$ \\
\hline 998 & $1.35 \mathrm{E}-03$ & $3.42 \mathrm{E}-03$ & 4.75E-04 & $4.93 \mathrm{E}-05$ & $2.65 \mathrm{E}-03$ & $9.54 \mathrm{E}-04$ & $2.65 \mathrm{E}-05$ & $1.47 \mathrm{E}-05$ & $8.39 \mathrm{E}-06$ & $1.19 \mathrm{E}-04$ & $5.07 \mathrm{E}-05$ \\
\hline 1023 & $1.95 \mathrm{E}-04$ & $3.14 \mathrm{E}-03$ & $3.75 \mathrm{E}-04$ & $3.12 \mathrm{E}-05$ & $3.09 \mathrm{E}-03$ & $1.39 \mathrm{E}-03$ & 2.14E-05 & $1.30 \mathrm{E}-05$ & $1.68 \mathrm{E}-05$ & $1.51 \mathrm{E}-04$ & $1.31 \mathrm{E}-04$ \\
\hline 1048 & $2.49 \mathrm{E}-05$ & $2.79 \mathrm{E}-03$ & $2.36 \mathrm{E}-04$ & $2.94 \mathrm{E}-05$ & $3.37 \mathrm{E}-03$ & $1.79 \mathrm{E}-03$ & $1.59 \mathrm{E}-05$ & $1.22 \mathrm{E}-05$ & $2.75 \mathrm{E}-05$ & $1.31 \mathrm{E}-04$ & $1.94 \mathrm{E}-04$ \\
\hline
\end{tabular}




\begin{tabular}{cccccc}
\multicolumn{7}{c}{ FTIR Data } \\
\hline $\mathbf{T ~}(\mathbf{K})$ & HCl & Methane & CO & $\mathbf{C O}_{2}$ & Formaldehyde \\
\hline 573 & 0 & 0 & 0 & 0 & 0 \\
673 & 0 & 0 & 0 & 0 & 0 \\
723 & 0 & 0 & 0 & 0 & 0 \\
873 & $7.18 \mathrm{E}-05$ & 0 & $4.72 \mathrm{E}-04$ & 0 & $2.37 \mathrm{E}-05$ \\
923 & $1.27 \mathrm{E}-03$ & $5.45 \mathrm{E}-05$ & $2.09 \mathrm{E}-03$ & 0 & $1.05 \mathrm{E}-04$ \\
948 & $2.24 \mathrm{E}-03$ & $4.28 \mathrm{E}-04$ & $4.08 \mathrm{E}-03$ & $9.57 \mathrm{E}-06$ & $7.45 \mathrm{E}-04$ \\
973 & $2.98 \mathrm{E}-03$ & $1.32 \mathrm{E}-03$ & $7.56 \mathrm{E}-03$ & $1.41 \mathrm{E}-04$ & $1.08 \mathrm{E}-03$ \\
985 & $3.44 \mathrm{E}-03$ & $1.68 \mathrm{E}-03$ & $9.70 \mathrm{E}-03$ & $3.05 \mathrm{E}-04$ & $7.23 \mathrm{E}-04$ \\
998 & $3.67 \mathrm{E}-03$ & $2.71 \mathrm{E}-03$ & $1.28 \mathrm{E}-02$ & $5.52 \mathrm{E}-04$ & $5.01 \mathrm{E}-04$ \\
1023 & $3.67 \mathrm{E}-03$ & $3.50 \mathrm{E}-03$ & $1.39 \mathrm{E}-02$ & $8.06 \mathrm{E}-04$ & $1.12 \mathrm{E}-04$ \\
1048 & $4.16 \mathrm{E}-03$ & $4.08 \mathrm{E}-03$ & $1.58 \mathrm{E}-02$ & $1.15 \mathrm{E}-03$ & $4.42 \mathrm{E}-06$ \\
\hline
\end{tabular}

